10th International Symposium on Process Systems Engineering - PSE2009

Rita Maria de Brito Alves, Claudio Augusto Oller do Nascimento and Evaristo

Chalbaud Biscaia Jr. (Editors)

(C) 2009 Elsevier B.V. All rights reserved.

\title{
Feasibility of separation of ternary mixtures by pressure swing batch distillation
}

\author{
Gabor Modla, Arpad Kopasz, Peter Lang \\ BUTE Department of Building Services and Process Engineering, Muegyetem rkp. 3-5 \\ H-1521 Budapest, Hungary, E-mail:lang@mail.bme.hu
}

\begin{abstract}
Feasibility of the pressure swing batch distillation separation of ternary homoazeotropic mixtures in different single and double column configurations is investigated by assuming maximal separation. Feasibility regions where the ternary mixture can be separated into its pure components (by applying in at least one step pressure swing) and the separation steps for different configurations are determined. The method is presented for the most frequent types of ternary mixtures with minimum azeotrope(s).
\end{abstract}

Keywords: Pressure Swing, Batch Distillation, Azeotropes

\section{Introduction}

Azeotropic mixtures cannot be separated into their components by traditional distillation. Several mixtures form a homoazeotrope, whose position can be shifted substantially by changing system pressure (pressure sensitive azeotropes). These mixtures can be separated by pressure swing distillation (PSD). Knapp et al. (1992) studied the use of entrainers for continuous PSD. Hence such azeotropes can be separated by PSD which are not pressure sensitive if the entrainer forms a pressure sensitive boundary and the number of mixtures separable by PSD can be increased. Phimister and Seider (2000) studied the separation of a minimum azeotrope by semicontinuous PSD. Repke et al. (2007) investigated experimentally the application of PSD in batch. They studied the separation of a minimum azeotrope (acetonitrile-water) by PSD in a batch rectifier (BR) and in a batch stripper (BS). Modla and Lang (2008) compared different batch configurations for the separation binary (maximum and minimum) homoazeotropes by PSD. The best results were obtained with the two new double column configurations (double column batch rectifier (DCBR) for maximum and double column batch stripper (DCBS) for minimum azeotropes) equipped with a common bottom or top vessel, respectively.

Modla et al. (2008) stated that by the methods of classification of Serafimov (1970) and Matsuyama and Nishimura (M\&N, 1977)) the pressure sensitivity of an azeotrope is not indicated though this information is indispensable for the synthesis of PSD separations. They extended the method of M\&N by indicating the pressure sensitivity of an azeotrope by writing ' $\mathrm{P}$ ' after the $\mathrm{M} \& \mathrm{~N}$ number of an azeotrope if it is pressure sensitive. (They also mentioned that if the type of RCM varies (an azeotrope disappears or the volatility order of components varies with the variation of pressure), it must be given for both pressures.)

The aim of our work is to investigate the feasibility of the batch PSD separation of ternary mixtures in different (single and double) configurations by assuming maximal separation, to determine feasibility regions where the ternary mixture can be separated 
into its pure components (by applying in at least one step pressure swing) and to determine separation steps for ternary azeotropic mixtures of different type.

\section{Feasibility method}

When making feasibility studies we suppose that maximal (perfect) separation (Bernot et. al. 1990) can be produced. This involves the following assumptions: 1) large number of stages $(N), 2$ ) large reflux/reboil ratio, 3) negligible liquid plate hold-up, 4) negligible vapour hold-up.

The method is based on the determination of the feasible compositions of products (continuously withdrawn) and those of residues (remaining in the vessel) by analysing the vessel paths in the residue curve maps at the two different pressures $\left(P^{1}, P^{2}\right)$.

For the different column configurations separation steps and feasibility region(s) of the separation, which means all feed compositions, from where all components can be purely recovered by maximal separation at the given pressure or by applying pressure swing, are determined.

In order to determine the sequence of the cuts (products) for a given charge composition by maximal separation we have to study the batch distillation (BD) regions. From any point of a $\mathrm{BD}$ region we get the same cuts (fractions) by rectification. The method to divide the composition diagram into BD regions can be summarised as follows:

1. First the composition triangle is divided into regions containing one unstable node.

2. In each of these regions the other batch distillation boundaries are found by joining the unstable node to all the saddles and nodes of its region.

Since we make feasibility studies not only for BRs but also for batch strippers we have to define also the batch stripping (BS) region: from any point of a BS region we get the same cuts by stripping. In the case of strippers the maximal separation means large reboil ratio (and $N$ ). In order to distinguish better the BD regions (used for rectification) and $\mathrm{BS}$ ones in this paper the BD regions will be called batch rectification (BR) regions. In a ternary mixture containing pressure sensitive azeotrope(s) the location of the boundaries involving this (these) azeotrope(s) varies. (We suppose that the boundaries are straight lines.)By changing the pressure we can cross these boundaries. Between the boundaries concerning to the two different pressures there is a region from where at the different pressures different products (distillate in a BR, bottoms in a BS) can be obtained.)

\section{Feasibility results}

Results will be shown for the two most frequent Serafimov's types of mixture, only. Sketch of the residue curve map (RCM), simple distillation, BR/BS regions, separation steps and vessel-paths for the recommended configuration(s) are presented.

\subsection{Separation of mixtures of class 1.0-1a}

The classification of original residue curve map is 1.0-1a (21.6\%) by Serifamov (S) and it is $1-0-0$ by $N \& M$.

The azeotrope $L-I$ is the unstable, vertex $H$ is the stable node, respectively (Fig. 1a). Vertices $L$ and $I$ are saddles. The RCM (M\&N: 1P-0-0, e.g. CCl4-ethanol+acrylic acid) contains only one simple distillation (the whole triangle) but two BR and BS (maximal separation) regions. For this type of mixture the BR and BS regions coincide but the sequence of the products (cuts) is just the opposite as it is indicated in Table 1 where sequence of the vertices of the different regions (triangles) corresponds to that of the cuts. 
The location of the boundary (line between $A z_{L I}$ and vertex $H$ ) considerably varies and so the areas of the BR and BS regions change with the pressure.

Configurations recommended: batch stripper or double column batch stripper.

The separation steps are as follows:

1. Production of $H$ :

In the case of DCBS only one column is operated; at the end of this step the vessel path (Fig. $1 b$ ) arrives at the $L I$ edge. This step is feasible at either of the two pressures but if the charge composition is located

- in the area I and $P^{2}$ is applied or

- in the area II and $P^{1}$ is applied

there is no need for pressure change at the end of this step.

2. Production of $L$ and $I$ by pressure swing distillation (in the case of DCBS both columns are operated).

Remarks:

-The separation of the ternary mixture can be reduced to that of binary systems.

-This type of ternary mixture cannot be separated in one operation step by double column system.

The feasible region is the whole area of the triangle.

\begin{tabular}{|l|l|l|}
\hline Region & I. & II. \\
\hline BR & $A z_{L I}-I-H$ & $A z_{L I}-L-H$ \\
\hline BS & $H-I-A z_{L I}$ & $H-L-A z_{L I}$ \\
\hline
\end{tabular}

Table 1. BR and BS regions for a mixture 1-0-0

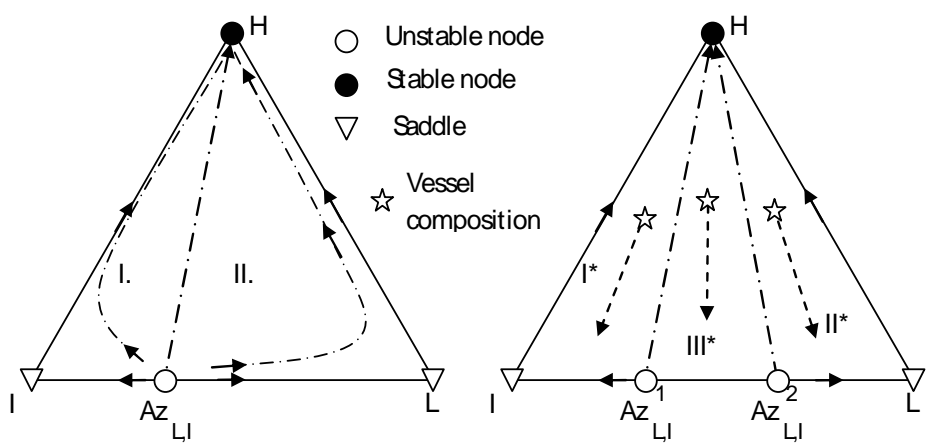

Fig. 1. Sketch of the RCM (a) and vessel paths of a BS for a mixture 1P-0-0

3.2. Separation of mixtures $2.0-2 b$

The class of original residue curve map is $2.0-2 \mathrm{~b}(21 \%)$ by Serifamov and it can be 1-20 or $1-0-2$ or $0-2-1$ by N\&M.

The azeotrope $I-H$ and vertex $L$ are saddles, vertices $I$ and $H$ are stable nodes and the azeotrope $L-I$ is the unstable node, respectively (Fig. 2). The RCM contains two simple distillation and three BR/BS regions (Table 2).

If only one azeotrope is pressure sensitive it can be - the unstable node, (by extended classification of RCM: 1P-2-0 (Case1) or 1P-2-0 or 02-1P) or - a saddle (by extended classification of RCM: 1-2P-0 1-0-2P, 0-2P-1; not shown). The other possibility is that both azeotropes are pressure sensitive (Case 2). 


\begin{tabular}{|l|l|l|}
\hline Region & I. & II. \\
\hline BR & $A z_{L I}-I-H$ & $A z_{L I}-L-H$ \\
\hline BS & $H-I-A z_{L I}$ & $H-L-A z_{L I}$ \\
\hline
\end{tabular}

Table 2. BR and BS regions for a mixture 1-0-0
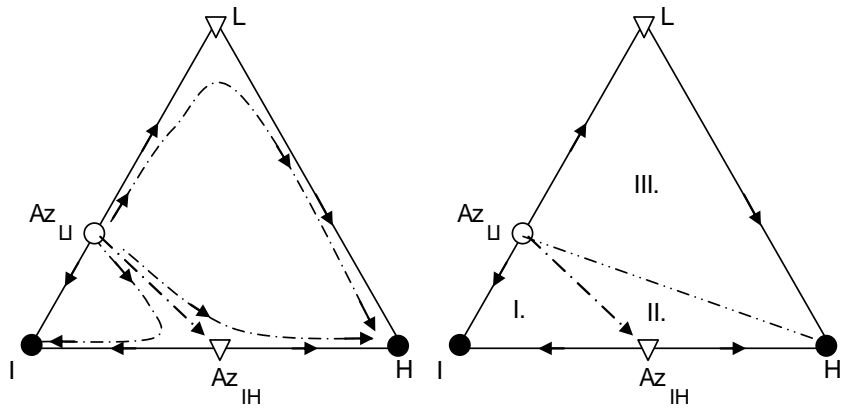

Fig. 2. Sketch of RCM (a) mixture and BR/BS regions (b) for a 1-2-0 mixture (2.0-2b)

3.2.1. Case 1 - Only one azeotrope is pressure sensitive

The pressure sensitive azeotrope is the unstable node (e.g. Az $z_{L I}$, Figs. 3)

The location of the boundary (line between $A z_{I H}$ and $A z_{L I}$ ) considerably varies with the pressure (due to the movement of $A z_{L I}$ ) .

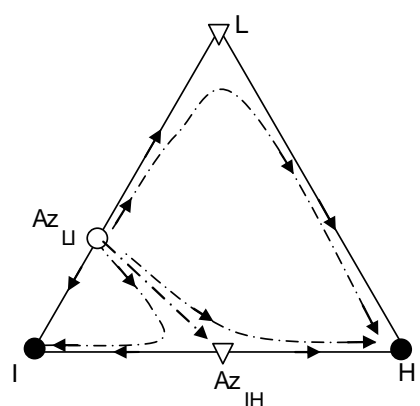

a. Sketch of RCM at $P 1$

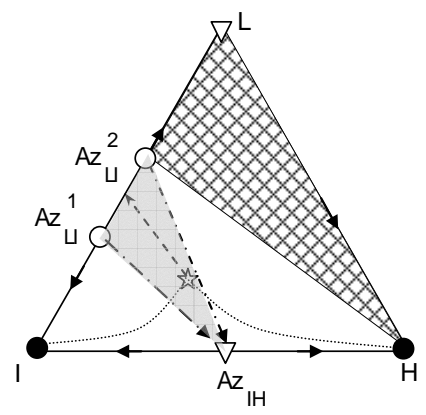

c. Vessel path for the DCBS

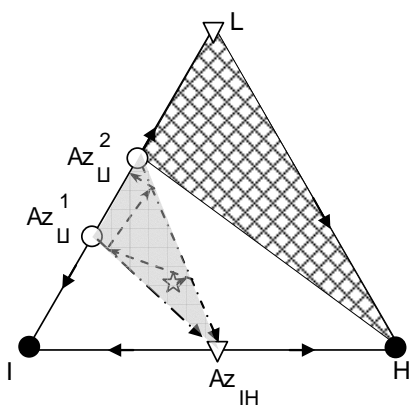

b. Vessel path for the PS-BS

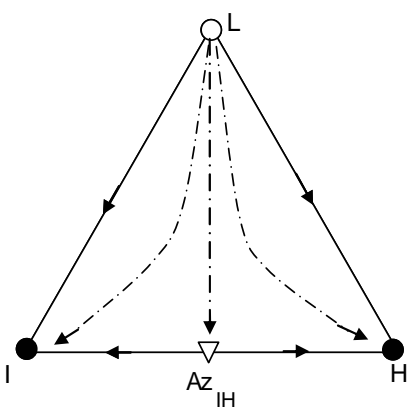

d. Sketch of RCM at P3

Fig.3. Separation of 1P-2-0 mixtures 
The configurations recommended: batch stripper (Fig. 3b) or double column batch stripper (Fig. 3c).

The separation method contains the following steps:

0 . (optional). Production of some $I$ or $H$ : in the case of DCBS by operating only one column, if the charge composition is out of the darkened triangle.

1. Production of $H$ or $I$ alternately in a BS, (vessel path (dashed) in Fig. 3b) or simultaneously in a DCBS (Fig. $4 c$ the liquid concentration profiles are dotted)). (In the case of BS this step consists of several parts and requires several changes of $P$.)

2. Production of $L$ and $I$ by PSD.

If the charge composition is located in the BS Region III (hatched area for $P^{2}$ in Fig. $3 b$ ) in the first production step the whole amount of $H$ can be withdrawn as product.

It must be still noted that with the variation of the pressure (e.g. at $P^{3}$ ) the azeotrope $L-I$ can even disappear (Fig. 3d). In this case the topology of the residue curve map varies (to Class S: 1.0-2, M\&N: 0-2-0). The recommended configurations are the same as above. In this case the separation $L / I\left(\right.$ at $P^{3}$ ) does not require pressure swing. The feasible region is the whole area of the triangle.

\subsubsection{Case 2 - Both azeotropes are pressure sensitive}

In this case there are two boundaries whose location varies with the pressure. We have to focus on the variation of the concentration of that component (e.g. component $I$ in Fig. 4) which is present in both azeotropes. Two different cases are possible. The concentration of that component in the two azeotropes can vary with the pressure

- in the same direction (increases or decreases (Fig. 4c) in both azeotropes)

- in the opposite direction when the boundaries intersect.

Recommended separation methods:

1. Removal of $\mathrm{Az}_{L I}$ by $\mathrm{BR}$ which is pressure sensitive so it can be separated by pressure swing BS or DCBS (Fig. 4a). The residue can be binary mixture $I-H$ or $L-H$. The mixture $L-H$ is zeotropic so it can be separated by both BR and BS while the mixture I$\mathrm{H}$ can be separated by pressure swing in BS or DCBS.

2. If the charge composition is located in the BS region III (the hatched area in Fig. 4b) the first step is production of $H$ in BS. In this case the remaining binary mixture $L-I$ which can be separated by pressure swing in BS or DCBS.

The feasible region is the whole area of the triangle.

The most frequent types of ternary mixtures were investigated. We studied 5 types of mixture by the classification of Serafimov corresponding to 11 different types of mixture by the classification of Nishimura and Matsuyama (N\&M), and 41 different cases by the extended N\&M classification taking into account the pressure sensitivity of the azeotropes, as well. From these 41 cases studied

-in 14 cases the whole area of the composition triangle proves feasible,

-in 15 cases in the triangle there is at least one region where the separation is feasible,

-in 12 cases there is no feasible area within the interior of the triangle.

We stated that, the composition triangle contains a feasible part only if

- both the azeotrope which is unstable node and

- the separatrice(s) between stable node(s) and saddle(s)

are pressure sensitive.

Moreover we concluded that if the cuts of a BR/BS region are only pure component(s) and/or pressure sensitive binary azeotrope(s) this region is feasible. 


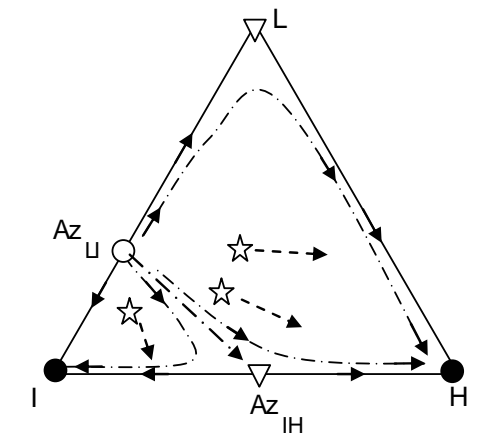

a. Feasible still paths in a $B R$

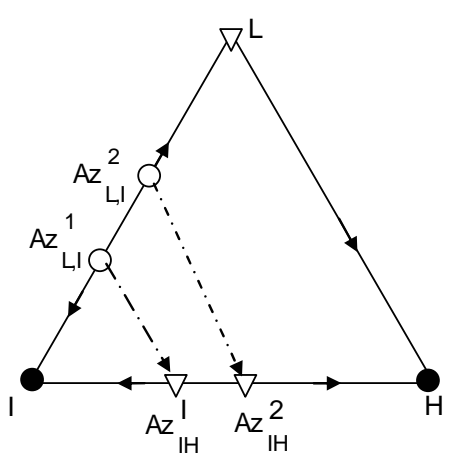

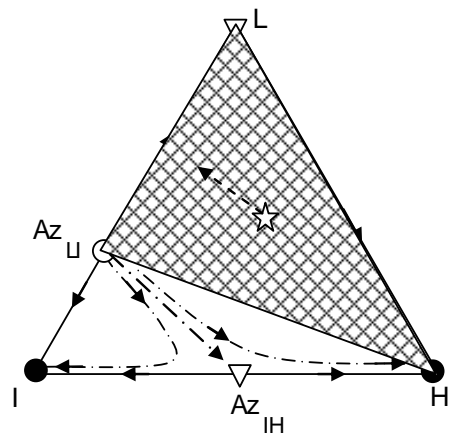

b. A feasible top vessel path in a BS

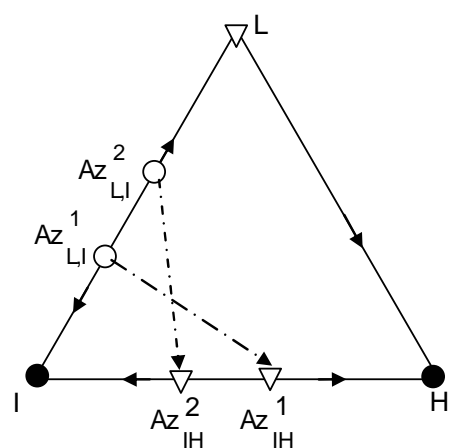

c. Movement of the boundaries with $P$ in the same direction $d$. in the opposite direction

Fig. 4. Separation of $1 P-2 P-0$ mixtures:

\section{Acknowledgement}

This paper was supported by the Janos Bolyai Research Scholarship of the Hungarian Academy of Sciences and the Hungarian Research Funds (OTKA) (No: T-049184).

\section{References}

Bernot Ch., M. Doherty, M. F. Malone, (1990). Patterns of Composition Change in Multicomponent Batch Distillation, Chem. Eng. Sci., 45, 1207

Knapp J.P. and M.F. Doherty, (1992). A new pressure swing-distillation process for separating homogeneous azeotropic mixtures, Ind. Eng. Chem. Res., 31, 346-357.

Matsuyama, H., H. Nishimura, (1977). "Topological and Thermodynamic Classification of Ternary Vapor-Liquid Equilibria", J. Chem. Eng. Japan, 10, 181.

Modla G. and Lang P. (2008). Feasibility of new pressure swing batch distillation methods, Chem. Eng. Sci., 63 (11) 2856-2874.

Modla G., P. Lang and A. Kopasz, (2008) Entrainer selection for pressure swing batch distillation, ESCAPE-18, in CD, ISBN 978-0-444-53228-2.

Phimister, J.R.; Seider, W.D. (2000). Semicontinuous, Pressure Swing Distillation, Ind. Eng.Chem. Res., 39, 122-130.

Repke .J. U., Klein A., Bogle D., Wozny G., (2007). Pressure Swing Batch Distillation for Homogenous Azeotropic Separation, ChERD 85 (4) 152, 492-501

Serafimov, L.A. (1970). The Azeotropic Rule and the Classification of Multicomponent Mixtures VII. Diagrams for Ternary Mixtures. Russ. J. Phys. Chem. 44(4), 567-571 